\title{
Fatal rupture of an unsuspected post-traumatic aneurysm of the thoracic aorta during pregnancy
}

\author{
J N Townend, M K Davies, E L Jones
}

University of Birmingham Department of Cardiovascular Medicine, Queen Elizabeth Hospital, Birmingham JN Townend M K Davies

University of Birmingham Department of Pathology, Medical School, Birmingham E L Jones

Correspondence to Dr J N Townend, Department of Cardiovascular Medicine, Queen Elizabeth Hospital, Queen Elizabeth Hospital Edgbaston,
B15 2TH.

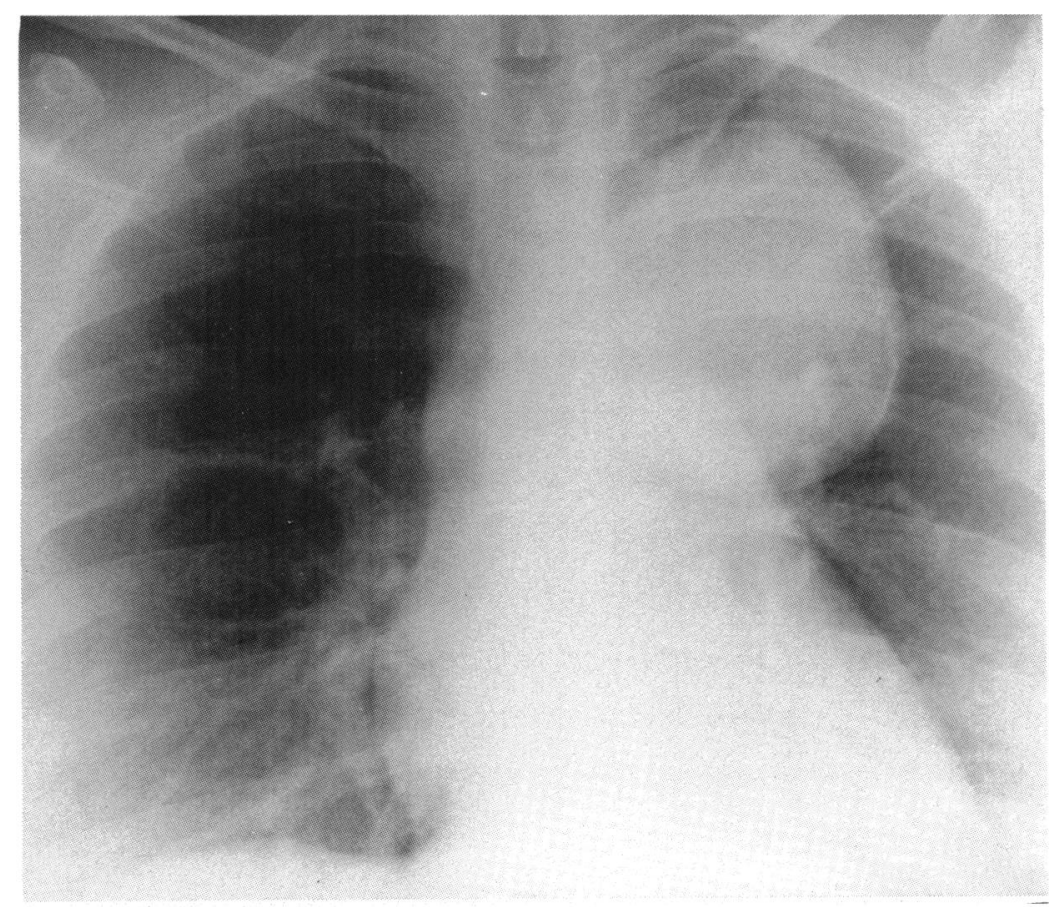

Chest radiograph showing a smooth rounded mass arising from the left upper mediastinum with calcification in the lateral aspect.

\begin{abstract}
A 27 year old woman presented during the third trimester of her second pregnancy complaining of chest pain. A chest radiograph showed a large aneurysm arising from the distal part of the aortic arch. She was transferred to the regional cardiothoracic centre but died suddenly on arrival. Necropsy confirmed a ruptured saccular aortic aneurysm arising just distal to the left subclavian artery. $A$ history of a road traffic accident at the age of 12 was obtained from relatives after the patient's death.

Vascular and haemodynamic changes in pregnancy may have caused the rupture of this previously silent posttraumatic aortic aneurysm.
\end{abstract}

Rupture of arterial aneurysms is a rare complication of pregnancy. ${ }^{1}$ Almost all of the reported cases of rupture of thoracic aortic aneurysms during pregnancy have been dissecting aneurysms; saccular aneurysms of the aortic arch are rare in young women and are usually secondary to systemic disease. We

report a case of fatal rupture of a saccular thoracic aortic aneurysm during pregnancy in a previously fit young woman with no evidence of systemic disease. A history of previous trauma was obtained only after she died.

\section{Case report}

A 27 year old woman presented to her local hospital at 38 weeks' gestation in her second pregnancy complaining of chest and interscapular pain of 3 hours' duration. The pain had started suddenly during a visit to a supermarket and was associated with a "numb" sensation in the left arm. There was no prior history of chest pain or cardiopulmonary disease. Antenatal clinic visits had revealed mild hypertension at 34 weeks (blood pressure 140/ $96 \mathrm{mmHg}$ ) but otherwise the pregnancy had been uneventful. Routine serological testing for syphilis was negative. The first pregnancy two years earlier had also been complicated by mild hypertension; no chest radiograph was performed. She was obese and hypertensive with a blood pressure of $145 / 110 \mathrm{~mm} \mathrm{Hg}$ but no other abnormality was detected. A chest radiograph (figure) showed a smooth rounded mass arising from the left upper mediastinum with calcification in the lateral aspect. The left costophrenic angle was clear. An unstable thoracic aortic aneurysm was diagnosed and the patient was transferred by ambulance to our hospital, a distance of nearly 60 miles. Immediately on arrival the patient collapsed with no pulse or spontaneous respiration: all efforts at resuscitation were unsuccessful. During the resuscitation procedure an emergency ceasarean section was performed but the infant was born with an Apgar score of zero and died several hours later.

A large left haemothorax was found at necropsy. The heart was normal as were the ascending aorta and the innominate, left common carotid, and left subclavian arteries. Immediately distal to the origin of the left subclavian artery there was a slight narrowing of the aorta distal to which there was a saccular aneurysm measuring $7 \times 7 \mathrm{~cm}$. The walls were very thin with foci of calcification and there was a posterior tear communicating with the left hemithorax. The rest of the aorta and arterial system was normal; there was no evidence of intimal fibrosis, medionecrosis, or dissection. Microscopy showed that the wall of the aneurysm was composed of thin layers 
of dense hyalinised fibrous tissue with foci of calcification and cholesterol crystal clefts consistent with old haemorrhage. The outer media and adventitia were intact. The aortic media at the constriction adjacent to the neck of the aneurysm showed fibrous disruption of the elastic lamellae consistent with an old traumatic laceration. Elsewhere the aortic wall was normal.

Relatives were subsequently asked about any history of injury. One recalled that at the age of 12 the patient had been a passenger in a car that overturned. She had been apparently unhurt and did not seek medical attention. It seems likely that a deceleration injury sustained then caused an aortic tear at the typical site and led to the development of a posttraumatic aneurysm.

\section{Discussion}

Sudden death owing to rupture of a posttraumatic aneurysm of the thoracic aorta during pregnancy has not been reported before. However, a case resembling this one was reported by Locufier $e t$ al in 1989. ${ }^{2}$ They described a 22 year old woman in whom a saccular aortic aneurysm almost identical in position and size to our case was discovered on a "routine" chest radiograph performed the day after delivery. Pregnancy had been complicated by a preeclamptic episode but no other causative factor was identified. The aneurysm was successfully repaired.

Over $95 \%$ of traumatic aortic injuries involve the thoracic aorta and the usual site of laceration is 2 to $3 \mathrm{~cm}$ distal to the origin of the left subclavian artery. The adventitia can remain intact after the tearing of the inner layers of the aortic wall and this may lead to the development of a post-traumatic aneurysm. The inner margins of such aneurysms are formed of intimal fibrous strictures which may resemble a coarctation. ${ }^{3}$ Only $2-5 \%$ of patients with aortic rupture survive long enough to develop a chronic aneurysm ${ }^{4}$ and the natural history is that of progressive enlargement and eventual rupture. However, nearly one fifth of patients remain symptom free for over ten years after the causative injury. ${ }^{5}$ It seems that in our patient rupture was precipitated by pregnancy and associated hypertension 15 years after the presumed injury.

Rupture of arterial aneurysms is a rare but recognised complication of pregnancy. ${ }^{1}$ Changes in haemodynamic function (increases in blood pressure, stroke volume, and rate of pressure rise in systole) as well as in the structure of the media and intima of arterial walls ${ }^{67}$ predispose to aneurysm formation and rupture. While intimal changes reverse after pregnancy those in the media do not, suggesting that multiparity may be a risk factor. ${ }^{8}$ Dissecting aneurysms are by far the most common form of aortic aneurysm presenting during pregnancy ${ }^{1}$ but saccular aneurysms have been described. ${ }^{9}$

Rupture of the cerebral, coronary, renal, ovarian, and in particular the splenic vessels during pregnancy have all been reported. ${ }^{1}$ The third trimester is the most common time of rupture of aortic (and other) aneurysms in pregnancy with $75 \%$ of cases occurring in this period compared with $15 \%$ in the second trimester. ${ }^{9}$ In most patients there seems to be no underlying cause other than pregnancy itself; previous trauma may be an unrecognised cause in an unknown proportion of patients. In an extensive historical review of aortic aneurysms presenting during pregnancy only one of 29 cases of aortic saccular aneurysms presenting during pregnancy was thought to be traumatic in origin; in 17 cases no aetiological factor other than pregnancy was identified.

The chest radiograph has been abandoned as a screening investigation for women in early pregnancy. There may be a case for its selective use in patients with a history of serious trauma, however remote, but we are doubtful that such a history would have been forthcoming in this case. In the absence of any screening test, only a high index of suspicion leading to rapid diagnosis after presentation and prompt referral for surgery offer any hope in this life threatening complication of pregnancy.

JNT is a British Heart Foundation Junior Research Fellow.

We thank Mr DER Bateman, Mr RS Bonser, and Dr RM Whittington, HM Coroner for Birmingham and Solihull Districts, for their permission to report this case.

1 Barret JM, Hooydonk JE, Boehm FH. Pregnancy-related rupture of arterial aneurysms. Obstet Gynecol Surv rupture of arter

2 Locufier JL, Bosschaerts T, Barthel J, Delwarte D, Barroy JP. Aneurysm of the descending thoracic aorta in a young woman. J Cardiovasc Surg 1989;30:499-502.

3 Kinley CE, Chandler BM. Traumatic aneurysm of thoracic aorta. A case presenting as coarctation. Can Med Assoc 1967;96:279-81.

4 Parmley LF, Mattingly TW, Manion WC, Jahnke EJ. Nonpenetrating traumatic injury of the aorta. Circulation 1958;17:1086-101.

5 Bennet DE, Cherry JK. The natural history of traumatic aneurysms of the aorta. Surgery 1967;61:516-23.

6 Manalo-Estrella P, Barker AE. Histopathological findings in human aortic media associated with pregnancy. Arch Pathol 1967;83:336-41.

7 Schwarz OH, Hawker WD. Hyperplasia and hypertension of uterine vessels during various stages of pregnancy. $\mathrm{Am}$ of uterine vessels during various

8 Wexler BC. Vascular degenerative changes in the uterine arteries and veins of mutiparous rats. Am J Obstet Gynecol 1970;107:6-16.

9 Pedowitz P, Perell A. Aneurysms complicated by pregnancy. Am J Obstet Gynecol 1957;73:720-35. 